\title{
Diet before and during pregnancy in the Southampton Women's Survey
}

\author{
S. R. Crozier, H. M. Inskip, K. M. Godfrey, C. Cooper, S. M. Robinson and the SWS Study Group \\ MRC Epidemiology Resource Centre, University of Southampton, Southampton General Hospital, \\ Southampton SO16 6YD, UK
}

The Southampton Women's Survey (SWS) has measured the diet, body composition, physical activity and social circumstances of 12583 non-pregnant women aged 20-34 years living in the city of Southampton, UK. Diet over the 3 months before interview was assessed using an interviewer-administered FFQ that records consumption of 100 food items. A total of 2057 women went on to become pregnant and provided further data in both early pregnancy ( 11 weeks of gestation) and late pregnancy ( 34 weeks of gestation). The same intervieweradministered FFQ was used to assess diet at all three time points. Principal component analysis was used to define dietary patterns. Women with high scores on the first component had diets in line with recommendations from the UK Department of Health and other agencies $^{(1,2)}$; this dietary pattern was termed the 'prudent' dietary pattern. Prudent diet scores were generated before pregnancy and in early and late pregnancy.

Intakes of twenty-one foods or food groups increased in early pregnancy, including white bread, breakfast cereals, cakes and biscuits, processed meat, crisps, fruit and fruit juices, dried fruit, sweet spreads, confectionery and hot chocolate drinks (all $P<0.0001$ ). Consumption of breakfast cereals, cakes and biscuits, processed meat, non-citrus fruit, sweet spreads and hot chocolate drinks increased further in late pregnancy (all $P<0.0001$ ). Whilst consumption of puddings, cream, milk, cheese, full-fat spread, cooking fats and salad oils, red meat and soft drinks did not change in early pregnancy, they increased in late pregnancy $($ all $P<0.001)$. The most marked increase was for breakfast cereals, from a median frequency of 4.5 times per week in early pregnancy to seven times per week in late pregnancy.

Intakes of eleven foods or food groups decreased in pregnancy. Consumption of rice and pasta, offal, salad vegetables, other vegetables, vegetable dishes, nuts, diet coke, wine, tea and coffee were lower in pregnancy than before pregnancy (all $P<0.0001$ ). Consumption of rice and pasta and offal were notably lower again in late pregnancy than in early pregnancy $(P<0.0001)$; the percentages of women consuming any offal were 48 before pregnancy, 22 in early pregnancy and 16 in late pregnancy. Consumption of green vegetables and boiled potatoes did not change in early pregnancy, but decreased in late pregnancy. The most marked decreases in early pregnancy were offal, wine and tea and coffee.

In terms of overall dietary patterns, prudent diet scores were slightly lower in early pregnancy than before pregnancy ( $-0.05 \mathrm{SD})$ and also in late pregnancy as compared with before pregnancy $(-0.06 \mathrm{SD})$. This finding reflects increases in consumption of white bread, cakes and biscuits, red and processed meat, crisps, confectionery, full-fat spread and soft drinks, alongside decreases in consumption of rice and pasta, vegetables and vegetable dishes and wine.

The SWS provides an important opportunity to understand current changes in diet into pregnancy that have not been described previously. Clear reductions in consumption of offal, wine and caffeinated drinks demonstrate an encouraging response to pregnancyrelated public health messages. However, the overall quality of diet, as assessed by the prudent diet score, did not improve in pregnancy. The importance of these changes is not yet understood. Ongoing work is evaluating the impact of these dietary changes on nutrient intake and examining their relationships with growth and development of the offspring.

1. Department of Health (1994) Nutritional Aspects of Cardiovascular Disease. London: H. M. Stationery Office.

2. Department of Health (1998) Nutritional Aspects of the Development of Cancer. London: The Stationery Office. 